This is a draft of an invited chapter that will be published by Oxford University Press in the forthcoming book Dimensions of Psychosis, edited by Carol A. Tamminga, Elena I. Ivleva, Ulrich Reininghaus, and Jim van Os, and due for publication in 2017.

\title{
Chapter G1. Symptom Network Models of Psychosis
}

\author{
Adela-Maria Isvoranu, MSc \\ Department of Psychological Methods, University of Amsterdam \\ isvoranu.adela@gmail.com \\ Lindy-Lou Boyette, PhD \\ Department of Clinical Psychology, University of Amsterdam \\ l.l.n.j.boyette@uva.nl \\ Sinan Guloksuz, MD, PhD \\ Department of Psychiatry and Neuropsychology, Maastricht University Medical Centre \\ Department of Psychiatry, Yale School of Medicine \\ sinan.guloksuz@maastrichtuniversity.nl \\ Denny Borsboom, PhD \\ Department of Psychological Methods, University of Amsterdam \\ dennyborsboom@gmail.com
}

*To whom correspondence should be addressed: Adela-Maria Isvoranu, Department of Psychological Methods, University of Amsterdam, Nieuwe Achtergracht 129-B, Office 0.40, 1018 WT Amsterdam, The Netherlands; e-mail: isvoranu.adela@gmail.com

Disorders within the psychosis spectrum are highly heterogeneous and multifactorial. ${ }^{1,2}$ Despite intensive research over the past century, the causes and pathogenesis of psychosis are still unclear, and no genetic marker has been consistently linked to developing a psychotic disorder. $^{3-5}$ In recent years, in an attempt to overcome this conundrum, the conceptualization of mental disorders as networks of interacting symptoms has gained considerable ground. $^{4,6}$ This conceptualization aligns well with practitioners' viewpoints as it focuses on concrete symptoms and their interrelations, rather than on abstract latent disorders or syndromes. ${ }^{7}$

Even though direct influences of one symptom on another are routinely observed in clinical practice (e.g., if a patient shows social withdrawal, this may soon lead to the patient displaying paranoid ideation and vice versa), in classical (psychometric) approaches to psychosis, which underlie most common psychometric practices in research, such direct influences are not modeled. Instead, symptoms are treated as passive psychometric indicators of a (set of) latent variable(s) - thus, it is assumed that symptoms are simply a result of the underlying disorder, rather than influencing each other., ${ }^{4,6}$ As a result, correlations between symptoms are in a nontrivial sense spurious: symptoms cluster together because of their common dependence on the disorder.

The assumption that correlations between symptoms arise from a common cause, which has been deemed problematic by both psychometricians and clinicians $^{4,7}$, has spurred the development of alternative psychometric approaches to mental disorders, in which symptoms are viewed instead as networks of mutually interacting components. Collectively, these lines of research have become known as the network approach to mental disorders. The centerpiece of the network approach is the idea that symptoms are active causal agents in producing disorder states, and that the study of their causal interaction is central to progress in understanding and treating mental disorders. 
This chapter aims to introduce the network approach to mental disorders in the context of psychotic symptomatology. We first discuss standard approaches to psychotic disorders, highlighting unresolved issues, and we then provide an introduction to network models of psychosis, with a focus on the general theoretical framework. We concentrate on how (environmental and genetic) risk factors can be understood from a network perspective and how they can be included in network models. We complete the chapter with a discussion on how network models can be integrated into treatment approaches.

G1.1 PSYCHOSIS: DISEASE MODEL VERSUS NETWORK MODEL

"For the past twenty years, we have been biding time and asserting that certain etiological information is just around the corner $^{9}$; we still wait ${ }^{10}$. A reconsideration of our basic strategies and fundamental assumptions may be in order." 11

The concept of schizophrenia, and generally the disorders within the psychosis spectrum, as a disease entity emerged over a century $\operatorname{ago}^{2}$ and it soon became a central (theoretical and applied) framework in the fields of psychiatry and clinical psychology. This framework is now known in mental health research and psychiatry as the disease model. ${ }^{4}$ The scientific terminology used by clinicians and researchers alike quickly aligned with this view, and to date idioms such as "suffering from schizophrenia" are used to indicate the presence of a mental disorder. ${ }^{6}$ In addition, comprehensive research has argued in favor of using the term disease when describing schizophrenia, as the term disease implies a discrete entity with a specific etiology and therefore better aligns with the clarity and heuristic value of the disease model. ${ }^{2}$ Today, the use of the term "schizophrenia" almost immediately implies disease entity and in spite of fuzzy and unreliable results, biological approaches still focus on reverse-engineering this hypothesized disease entity using case-controls paradigms. ${ }^{12}$

The disease model strongly relies on the assumption that the nature of symptoms and the interactions between symptoms are not themselves relevant. It is argued that their presence is simply a result of the underlying disorder and that both research and treatment should focus on the disorder per se (see Figure 1). One example of this line of thinking that was very influential in the past decades is the idea that mental disorders are in fact diseases of the brain - literally "brain disorders" -, the nature of which will be uncovered through neuroscientific investigation. ${ }^{13}$ Notably, it is not neuroscience per se that is the problem, but the reductionist taxonomy in which current standard (psychometric) approaches are grounded on. With this framework in mind, extensive research has focused on identifying psychological and biological essences of mental disorders ${ }^{4}$ and on identifying common causes that give rise to symptomatology. ${ }^{6}$ However, this quest has not been as successful as expected and perhaps unsurprisingly the underlying assumption that symptoms are interchangeable has proved problematic - to date few researchers and even fewer clinicians defend it. ${ }^{4,5,7}$ Unlike in a medical context, where symptoms point to a disorder, which can then be confirmed with further testing (e.g., blood testing, x-ray), there is no diagnostic tool for any mental disorder. ${ }^{14}$ The diagnosis is entirely based on the clinical manifestations, and the progress of a disorder is determined only by tracing the progress of the symptoms and global functioning. Within a clinical context the dynamics between the symptoms themselves therefore become of central interest and a causal structure such as Paranoid Ideation $\rightarrow$ Anxiety $\rightarrow$ Social Isolation can often be observed. Given that the disease model does not allow (neither theoretically nor statistically) for such direct causal relations between symptoms, recent years have seen an emergence of network models of mental disorders, in which disorders are presumed to arise from this exact causal interplay between symptoms instead of being the result of a common cause. This approach, which turns the disease model on its head because it proposes that interactions between symptoms are themselves the causes of disorder states, aligns well with clinical intuition and current findings in the field; as a result, it currently spurs many novel research approaches to the old questions of what mental disorders are and where they come from.

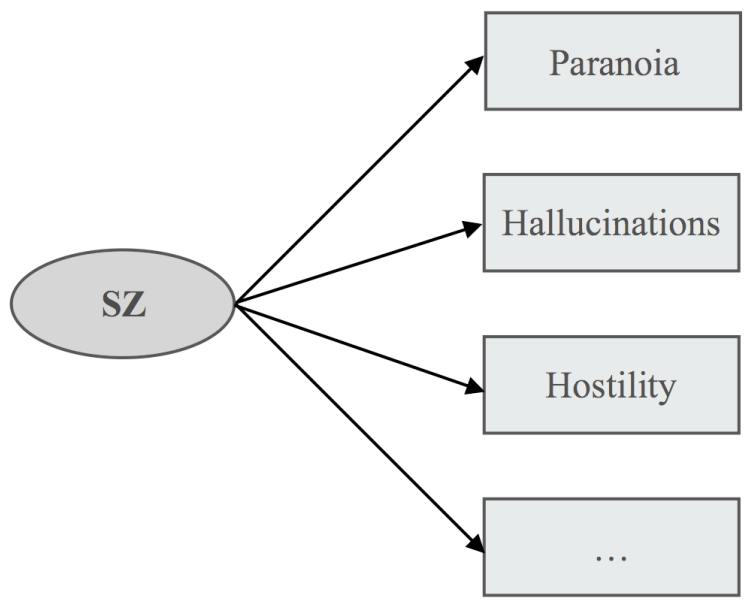

Figure G.1. (Simplified) visualization of the disease model for schizophrenia. According to this model, schizophrenia (SZ) is the root cause of its symptoms (three symptom examples as extracted from the Positive and Negative Syndrome Scale ${ }^{51}$ ), which co-occur only because they are all caused by the same disorder.

Following these developments, the hypothesis has been advanced that common cause mechanisms for mental disorders cannot be found because no such mechanisms exist. $^{6}$ This hypothesis has spread quickly through the research fields of psychology and psychiatry. In particular, it has been argued that symptoms may cluster together not only because of a shared origin, but because they have the power to directly trigger and influence each other. ${ }^{4,7}$ This 
novel theoretical framework became known as the network approach to psychopathology ${ }^{4}$ and the fundamental assumption underlying it is that mental disorders arise from a complex interplay between symptoms and psychological, biological, and sociological components ${ }^{15}$; this interplay can be captured in a network model (see Figure 2). To date, network models have been applied to a wide range of constructs, including depression ${ }^{16-23}$, post-traumatic stress disorder $^{24}$, psychosis ${ }^{25-28}$, autism ${ }^{29,30}$, social anxiety disorders $^{31,32}$, substance abuse ${ }^{33}$, personality ${ }^{34,35}$, quality of life research ${ }^{36}$, and the more general structure of psychopathology ${ }^{37-39}$.

While networks models are based on innovative statistical methodology and offer radically new opportunities for theory formation and mathematical simulation of mental disorders, the notion that symptoms influence each other has been well-known. In fact, such symptom-symptom interactions, transcending traditional diagnostic categories, have long been observed and acknowledged by clinical practitioners - network models just naturally accommodate it. ${ }^{40}$ Importantly, in contrast to the mysterious latent entities posited in conventional statistical models of mental disorders, interactions between symptoms are often mundane and well understood. For instance, if a person thinks they are being followed by someone who would like to harm them (i.e., present signs of paranoid ideation), this person may soon become too anxious to walk outside and to freely carry out daily activities (i.e., present signs of social isolation). This behavior could then in turn re-activate the initial paranoia, as the patient is not provided with any counterevidence of the paranoid belief, allowing it to be sustained. In this way, the symptoms enter a feedback loop in which they reinforce each other; such feedback loops are often identified and are central in clinical practice. If symptom interactions are sufficiently strong, or if the symptom network is sufficiently large, the system can then enter into a stable state of sustained symptom activation. This arises when the interactions between symptoms are such that the symptom network is able to maintain its own activation - a state that we phenomenologically recognize as a mental disorder.

To wit, within this framework, disorders are not "mere labels" or social constructions: a stable state of problem activation is as real as anything, and as such in the network approach mental disorders are explained, but not explained away. Importantly, however, in the network approach a disorder such as schizophrenia is no longer understood as a disease entity that explains the symptoms. Instead, it is an alternative stable state of a network that is constituted by the causal interactions between symptoms. It can be illuminating to think of this in terms of an ecosystem analogy. It is well known that ecosystems, such as lakes, have alternative stable states (e.g., a lake can be clear of turbid). ${ }^{41}$ These stable states are a function of a highly complex interplay between various components that together determine their overall state (e.g., the number of fish in the lake, the state of the algae population, the amount of nitrate in the soil, etc.). Importantly, the stable states of a lake are real: the lake is either clear or turbid as a matter of objective empirical fact. However, the stable state of the lake is not properly conceptualized as a latent disorder that "underlies" or "explains" the state of relevant components in the system: it refers to a global, emergent state of the lake that arises out of the interactions between the components in the system, rather than a mysterious unknown latent entity that is responsible for the aligned state of the system components as we may encounter it (e.g., turbid water, an increased algae population, fewer fish). In the same way, network approaches conceptualize disorders as emergent global properties of the symptom interactions: they are real, but do not signify a central underlying disorder in the form of a generally applicable pathogenic pathway.

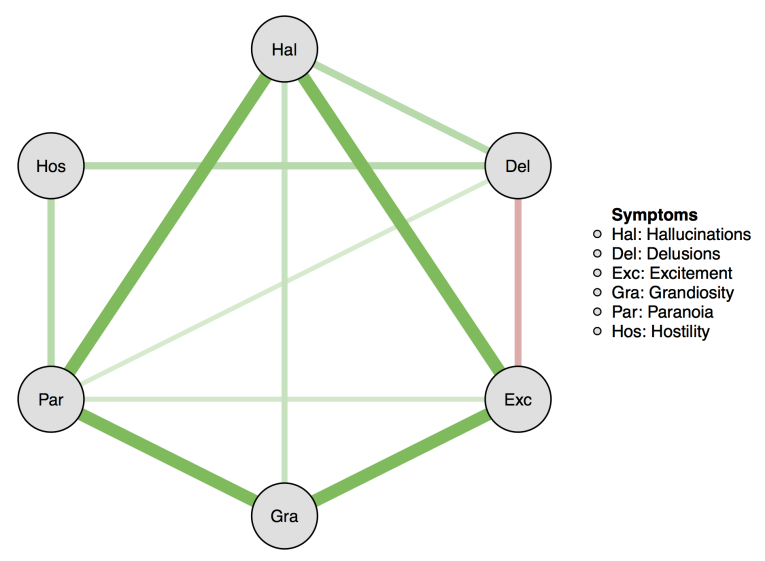

Figure G1.2. Visualization of a hypothetical network model of psychosis constructed using the qgraph ${ }^{42}$ package in the $R$ Statistical Software ${ }^{70}$. Green edges indicate positive associations, while red edges indicate negative associations between nodes. According to this model, the symptoms of schizophrenia (six symptom examples as extracted from Positive and Negative Syndrome Scale ${ }^{51}$ ) have causal power to trigger and influence each other.

Symptom interactions can be captured in a network structure (see Figure 2 for a hypothetical example of a network structure). Within such a network structure, symptoms are represented as nodes, while the relations between the symptoms are represented as edges. ${ }^{35,42}$ Notably, the nodes within a network do not have to be not limited to symptoms, but can also be, for instance, risk factors pertaining to the disorder (subchapter G1.2 details on how the influence of the genes and environment can be included in symptom networks), as well as non-symptomatic variables or protective variables. It is, however, important for network dynamics that these elements / variables can both influence other variables and be influenced by other variables (e.g., gender would not qualify here as it is immutable). ${ }^{40} \mathrm{~A}$ green edge indicates a positive relation between nodes, while a red edge indicates a negative relation between nodes ${ }^{42}$. For instance, if two nodes representing the 
typical symptoms anxiety and paranoid ideation are linked together by a green edge, this link indicates that the two nodes may positively activate each other (i.e., if a person is suffering from anxiety, the person is also most likely to present symptoms of paranoid ideation and / or vice versa). Likewise, if two nodes representing the typical symptoms excitement and blunted affect are linked together by a red edge, this link indicates that the two nodes may negatively activate each other (i.e., if a person is suffering from blunted affect, the person is also less likely to present symptoms of excitement and / or vice versa). Factors that impinge on the symptoms from outside together make up the external field of the symptoms. Note that in this case "from outside" means "from outside of the network" rather than "from outside of the person", so in addition to precipitating life events, drug use, or social circumstances, the external field can also include variable that relate to brain function and other bodily processes ${ }^{6}$. In the absence of a strong theory on the structure of these interactions, networks are commonly constructed from empirical data in an explorative fashion, by searching for the network structure that best explains the associations present in a dataset. ${ }^{4,35,42}$ Given the rapidly increasing popularity of these data-driven network models, it is important to note, however, that while there is a strong relation between network theory (which deals with causal interactions between symptoms) and statistical network models (which represent the statistical association between symptom measures and possibly other variables), that relation is by no means one-to-one. That is, many different statistical models could be used to inform a given network theory, and in some cases network theories are in fact constructed without any explicit statistical model; for example, by asking clinicians or respondents which symptoms activate each other. ${ }^{30,43,44}$

\section{G1.2 TOWARDS INCORPORATING ENVIRONMENTAL AND GENETIC RISK SCORES INTO NETWORK MODELS}

\section{2: "We are optimistically hopeful that the current mass of research on families of schizophrenics will discover an endophenotype, either biological or behavioral (psychometric pattern), which will not only discriminate schizophrenics from other psychotics, but will also be found in all the identical co- twins of schizophrenics whether concordant or discordant. All genetic theorizing will benefit from the development of such an indicator." 45}

Widespread research has focused on identifying biological and environmental risk factors involved in the onset and progression of schizophrenia. Despite such efforts, risk factors that cause psychotic manifestations remain elusive. $^{46,47}$ Meta-analytical work suggests that substance abuse (especially cannabis), growing up in an urbanized area, and developmental trauma are among the most common environmental influences that increase the susceptibility to psychosis ${ }^{47,48}$. Other factors include ethnicity, season of birth, prenatal stress and obstetric complications, but to a much lesser extent and with more inconsistent results ${ }^{46}$. In terms of a biological component, the look for biomarkers for schizophrenia has been extensive. However, to date, even though schizophrenia is known to be a highly heritable mental condition, with a rough heritability estimate up to $80 \%$, the search for candidate genes for schizophrenia has showed no robust findings. ${ }^{47}$ The diagnosis of schizophrenia is therefore based entirely on its clinical features and no other determinants have yet been incorporated into the diagnostic scheme. ${ }^{49}$ Notably, if all psychotic symptoms were indeed caused by an underlying disease entity - as schizophrenia is argued to be - one would expect to identify that symptoms have the same (or at least similar) risk factors (see Figure 3A). That is because risk factors are expected to only influence the liability to develop a disorder, while the disorder would, in turn, cause the symptoms. ${ }^{50}$ Current findings in the field of psychosis and depression suggest, however, that symptoms substantively differ in terms of their risk factors ${ }^{23,25,26}$, lending support to the importance of investigating the individual symptoms of a disorder and their causal associations - not only associations to each other, but also associations to the external field of influence.

In accordance, one can investigate the effects of the external field of influence (i.e., risk factors such as genes or environmental components) on a network structure in two ways. First, we can examine whether such factors have a direct main effect on the nodes (see Figure 3B, left panel). As highlighted in subchapter G1.2, nodes within a network are not restricted to symptoms only, but can be expanded to almost any variable - as long as the variable can both influence and be influenced by other variables ${ }^{40}$. As such, a node can represent any environmental, biological, or cognitive component that is deemed relevant for the disorder. To assess the main effect of these components we can construct a network that incorporates (1) environmental risk factors and symptoms, (2) genetic risk factors - such as a polygenic risk score - and symptoms, (3) both environmental risk factors and genetic risk scores and symptoms, depending on the availability of the data. In this way, we do not only examine whether symptoms differ in terms of their risk factors, but we can also identify associations between the risk factors themselves, including potential gene by environment interrelations. As network models are still novel, to date, we know of no studies that have incorporated polygenic risk scores into symptom networks; we are however aware of work in progress and expect extensive research to follow this avenue soon.

In terms of investigating the associations between environmental risk scores and individual symptoms of mental disorders, several studies in the field of depression $^{19,23,50}$ and psychosis ${ }^{25,26}$ have focused on assessing this interplay using network analysis. Isvoranu and colleagues $^{25,26}$ have identified that (1) when all symptoms of the Positive and Negative Syndrome Scale (PANSS) ${ }^{51}$ are included in a network alongside childhood trauma, general 
psychopathology symptoms appear to mediate the associations between trauma and the positive and negative symptoms; (2) when using general population data to examine the interrelations between environmental exposure - as measured by urbanicity, developmental trauma, and cannabis use - and psychopathology expression, the three environmental factors distinctly affect the symptomatology, with the main connective pathway being through cannabis use. In addition, strong associations between cannabis use and urbanicity, as well as between developmental trauma and cannabis use were identified ${ }^{26}$. Even though this line of research is in its early stages, it shows high promise in potentially unraveling means by which the external field influences symptoms. In short, with a network framework in mind, we convert from trying to understand and identify risk factors for schizophrenia to trying to identify risk factors for symptoms of schizophrenia and for the interaction between these symptoms.
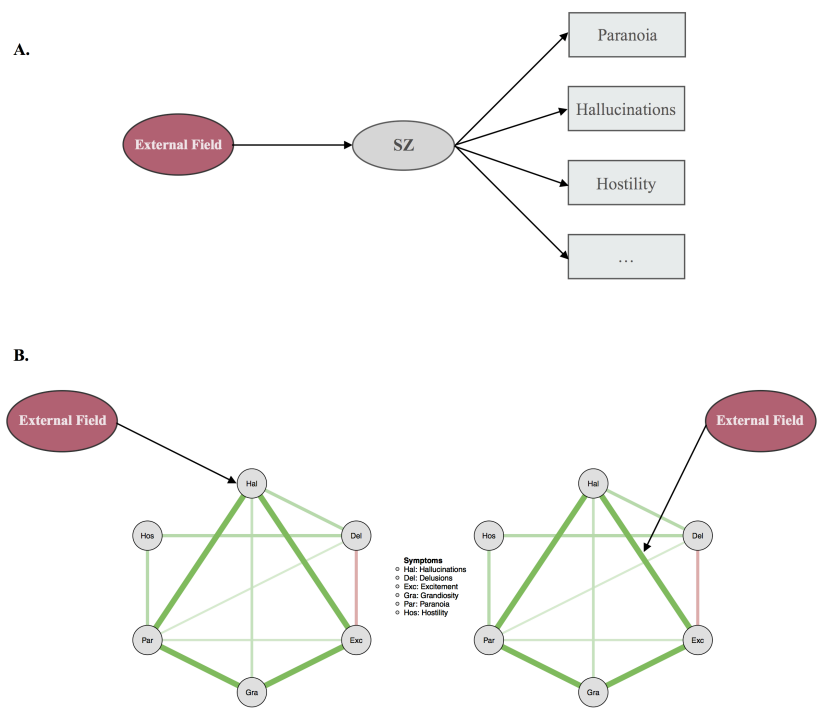

Figure G.1.3. A. Visualization showing how the external field (e.g., environmental risk factors, genetic risk scores) would be expected to influence symptoms from a common cause perspective. The influence takes place via the latent variable (schizophrenia), which then causes the symptoms. B. Visualization showing how the external field (e.g., environmental risk factors, genetic risk scores) would be expected to influence symptoms in network models. The influence takes place either as a main effect, on the node itself or as an indirect secondary effect, on the symptom-symptom interactions.

A second means (see Figure 3B, right panel) by which we can investigate the influence of the external field on symptomatology is through examining whether this has an indirect secondary effect on the network, by acting on the symptom-symptom connections. Thus, a stressor in the external field (e.g., grief, traumatic experiences, genetic components) cannot only be said to activate the network by either activating one or more symptoms (as detailed above), but also by increasing the strength of interactions between the symptoms themselves. Recent findings in large general population cohorts support this hypothesis - environmental exposure to risk factors such as childhood trauma, urbanicity, and cannabis use seem to alter the connectivity between the symptoms in a dose-response fashion, progressively triggering the transition towards a severe condition. ${ }^{52-55}$ Isvoranu and colleagues ${ }^{26}$ found that subjects exposed to cannabis showed increased connectivity between psychopathological dimensions such as depression and anxiety, as well as between interpersonal sensitivity and phobic anxiety, compared to subjects not exposed to any high-risk environmental factors. Correspondingly, Guloksuz and colleagues $^{53}$ showed using two general population cohorts that environmental exposure increased the degree of symptom connectivity within all symptom dimensions and related diagnostic categories, and further argued that the emergence of psychosis may be related to severity of increasingly connected psychopathology rather than to a specific illness category.

With these findings in mind, the influence of the external field as expressed through adverse environmental determinants seems to lead to a more strongly connected and thus less resilient symptom network, facilitating the transition to an alternative stable state of mental disorder. In particular, it may further be possible that part of the missing heritability issues faced at the moment are due to the fact that genetic vulnerability may be expressed as increased connectivity of symptom interactions rather than as a main effect on the network (or on the disorder). In addition, the impact of the environment and / or genetics does not appear to be specific to particular diagnosis categories, but vulnerability factors seem to rather be spread between the traditional diagnostic categories (e.g., childhood trauma ${ }^{56-59}$, cannabis use $\mathrm{e}^{60-62}$. Finally, thinking of this from a comorbidity perspective, if the external field has an influence on individual symptoms and the symptomsymptom interactions - which we know are often shared between disorders - the finding that vulnerability factors are similar and common between different diagnostic categories is no longer surprising.

\section{G1.3 CONCEPTUALIZING TREATMENT FROM A NETWORK PERSPECTIVE}

If a mental disorder indeed refers to a self-sustaining alternative stable state of a symptom network, as network theory holds, then this has direct implications for how to conceptualize treatment. To a considerable extent, existing treatments can be re-interpreted from a network perspective, and we may perhaps better understand the treatment mechanisms by looking at them in this way. Below, we give an overview of some thoughts and observations that may fit within this framework. It is also possible that, in the future, novel treatment protocols may be partly based on network theory; although we are still far removed from having operational network treatment, individualized network 
modeling has already been implemented in proof-of-concept studies $^{63}$, as we will also discuss.

As emphasized throughout this chapter, in a network model, there are basically three factors that determine its dynamics: the nodes in the network, the connections in the network, and the external field of factors outside of the network that influence it ${ }^{6}$. In accordance, we can consider three types of interventions from a network perspective; namely, those that target nodes in the network (henceforth: node-interventions), those that target connections in the network (henceforth: edge-interventions), and those that target the external field of factors outside of the network that influence it (henceforth field-interventions).

Assuming, for the moment, that we take the network to be the collection of symptoms that a patient with a psychotic disorder endorses, a node-intervention would be an intervention that is directly aimed at the reduction of a specific symptom. For instance, the use of an antipsychotic drug targeting derogatory auditory hallucinations may be considered an example of a node-intervention. In a similar fashion, a wide array of cognitive behavioral techniques that are aimed directly at challenging the content of a cognition could be considered node-interventions. For instance, consider a patient who believes that the voice that he or she hears originates from an outside source, but is willing to examine this belief. A behavioral experiment, a common technique in cognitive behavioral therapy, may be conducted, for instance asking the patient to tape the voice. Similarly, sleep reduction for reducing insomnia, responseprevention for compulsions, use of a drug with sedative qualities for agitation or interoceptive exposure for anxiety may all be considered examples of node-interventions.

Edge-interventions - interventions targeting relations between symptoms - may lie in various approaches in therapies that aim to teach a person how to improve cognitive, behavioral and affective coping skills that may impact symptom-symptom relations. For instance, in the case of derogatory auditory hallucinations, an edgeintervention may involve being taught a new adequate coping strategy by a clinician or support group, such as the use of distraction, humming, or setting limits and structure to the contact, instead of an unhelpful coping strategy, such as loudly arguing with the voices whenever they occur (for instance leading to agitation and/or social isolation due to social rejection). Quite recently, several new therapies that are specifically designed to increase general cognitive, behavioral and affective coping skills have gained ground for psychosis, such as Metacognitive Training (MCT) ${ }^{64}$ and Acceptance and Commitment Therapy (ACT) ${ }^{65}$. Metacognitive Training for psychosis aims to gain insight into unhelpful cognitive distortions putatively thought to be related to delusional beliefs (e.g. overconfidence in decisions, jumping to conclusions) and improve general metacognitive processes ('thinking about thinking'). Acceptance and Commitment Therapy aims, among others, to increase psychological flexibility, such as more openness with unwanted thoughts and feelings that are unlikely to be amenable to direct change strategies, and encourages valuedirected action. Instead of directly targeting symptoms when they occur, these types of interventions may decrease the connections, and therefore the disruptive impact of symptoms within a network.

Thirdly, field-intervention targets factors that are not directly part of the symptom network, but that do influence it from outside. For example, interventions include approaches that aim to restore or improve a person's community maintenance, such as entering a work project, which can reduce multiple symptoms and provide a buffer against different cascades of symptoms, and interventions that try to improve the physical environment of the person (e.g., by providing housing). Also interventions aimed at increasing the social support network of a person, or fighting the stigma of mental illness, may influence individuals symptom network from the outside.

We recognize that the distinction between types of interventions is not always clear-cut and do not argue that a 'direct' intervention cannot have multiple and indirect effects. The general aim of the above examples is to provide an idea of how existing interventions could be interpreted in a network theoretical framework. Currently, there is no way to utilize this information, but in the future one could think of systematic intervention planning that uses network models for the selection of (sequences of) interventions. In such cases, one would start with a (partly) personalized network model that captures the person's most important symptoms and interactions between them. One then asks: assuming a given set of node-, edge-, and field-interventions, what would be the most effective way of changing and charging the symptom network to repel it from its disorder state and create an enduring healthy attractor state? Network models could, in such a methodology, play the role of navigation software, in that they can suggest a number of routes that may result in effective treatment. Clearly, we have not even begun yet with seriously engaging such approaches, but in time network-based intervention planning may offer an attractive extension of the treatment professional's toolkit.

One of the problems that will have to be solved in order for this type of approach to work is that we need ways to assess a given person's network structure. There are currently two ways of approaching this problem, both of which are promising but also need considerable work before they can be effectively put to use. First, Frewen and colleagues $^{43}$ have developed so-called Perceived Causal Relations (PCR) scales. These scales first query which symptoms a person has, and then query the extent to which combinations of symptoms influence each other. One can administer these scales to clients themselves (resulting in a self-reported symptom network) or to others (e.g., family, friends, healthcare professionals), to get a qualitative idea of the most important connections between symptoms. The advantage of this methodology is that it is relatively fast (one can administer a PCR scale in less than an hour) and that it is straightforward to combine different sources of 
evidence (e.g., one can easily combine networks resulting from self-assessment and from assessment by others). The downside is that, if one wants to use the methodology to get a view of the actual symptom network, one has to assume that people can actually assess causal relations between symptoms. Although there is some evidence that people naturally represent disorders in this way ${ }^{44}$, it is not clear that this representation is also accurate.

A second way of getting a glimpse of symptomsymptom interactions is by using Ecological Momentary Assessment (EMA) protocols. In such approaches, which have seen tremendous growth in recent years due to the availability of smartphones, the person answers a short questionnaire multiple times a day. If sufficiently many datapoints are available, time-series analyses can be used to estimate the network structure of the interactions between queried symptoms ${ }^{66,67}$. In a proof-of-concept study, Kroeze and colleagues ${ }^{63}$ used this process to estimate a network structure for a number of clients in therapy; the network was subsequently shown to the client, and interventions were selected partly based on the network structure. Fisher and Boswell ${ }^{68}$ have pioneered similar treatment approaches using time series analyses, although they did not explicitly use network structures but instead based treatment on dynamic factor analysis models. The main advantage of these approaches is that they can uncover symptom-symptom interactions that people may not be able to identify themselves.

A promising future extension is that it will become possible to combine subjective assessments with more complex and objective behavioral and physical measures (e.g., movement, location, physiological data, etc.). However, it should be noted that current approaches suffer from a number of serious drawbacks that need to be addressed in future work: (a) data-analysis methods are currently suboptimal as they do not allow to model mean trends but only deviations from these trends, (b) there is no way to currently integrate processes that operate at different time scales (moments, hours, days, weeks), (c) many symptoms and external factors simply cannot be assessed using EMA, either for practical or for principled reasons. Discussing such limitations of the EMA approaches - as well as more general limitations of the network approach is beyond the scope of this chapter; for detailed theoretical and methodological considerations we direct the reader to the cited review articles ${ }^{40,69}$.

\section{CONCLUDING REMARKS}

The field of mental health care is in need of conceptualizations of pathology that are aligned with the complexity and multitude of symptoms and problems that most patients present with. Tools that could advance our understanding of psychiatric disorders, as well as provide substantiated intervention selection could be highly beneficial to clinical practice. In time, the network approach may provide such tools. Notably, it is unlikely that there is such a thing as a "one-size fits all treatment", especially given the inherent heterogeneity of psychosis. Thus, it is likely that intervention planning and monitoring will require personalized network models, which may vary from person to person. Endorsing integrative treatments approaches that are tailored to the individual, as well as embracing new avenues that allow us to combine subjective assessments with physical measures, will likely contribute to the advancement of the field and clinical practice. These possibilities are within reach, and in the next few decades we are bound to learn to what extent the promises of network theory in coordinating research and treatment will be fulfilled.

\section{REFERENCES}

1. Weinberger DR, Harrison PJ, eds. Schizophrenia. Oxford, UK: Wiley-Blackwell; 2010.

doi:10.1002/9781444327298.

2. Tandon R, Keshavan MS, Nasrallah HA.

Schizophrenia,"just the facts", what we know in 2008: Part 1. Overview. Schizophr Res. 2008;100(1-3):4-19.

doi:10.1016/j.schres.2008.01.022.

3. Tandon R, Keshavan MS, Nasrallah HA. Schizophrenia, "just the facts", what we know in 2008. 2. Epidemiology and etiology. Schizophr Res. 2008;102(1-3):1-18.

doi:10.1016/j.schres.2008.04.011.

4. Borsboom D, Cramer AOJ. Network analysis: an integrative approach to the structure of psychopathology. Annu Rev Clin Psychol. 2013;9:91-121. doi:10.1146/annurev-clinpsy-050212-185608. 5. Kendler KS, Zachar P, Craver C. What kinds of things are psychiatric disorders? Psychol Med. 2011;41(6):11431150. doi:10.1017/S0033291710001844.

6 . Borsboom D. A network theory of mental disorders. World Psychiatry. 2017;16(1):5-13.

doi:10.1002/wps.20375.

7. Fried EI. Problematic assumptions have slowed down depression research: why symptoms, not syndromes are the way forward. Front Psychol. 2015;6(March):309. doi:10.3389/fpsyg.2015.00309.

8. Schmittmann VD, Cramer AOJ, Waldorp LJ, Epskamp S, Kievit RA, Borsboom D. Deconstructing the construct: A network perspective on psychological phenomena. New Ideas Psychol. 2013;31(1):43-53.

doi:10.1016/j.newideapsych.2011.02.007.

9. Gottesman II, McGuffin P, Farmer a E. Clinical genetics as clues to the "real" genetics of schizophrenia (a decade of modest gains while playing for time). Schizophr Bull. 1987;13(1):23-47. doi:10.1093/schbul/13.1.23.

10. Sullivan PF. Schizophrenia genetics: the search for a hard lead. Curr Opin Psychiatry. 2008;21(2):157-160. doi:10.1097/YCO.0b013e3282f4efde.

11. Tandon R, Keshavan MS, Nasrallah HA.

Schizophrenia, "just the facts", what we know in 2008. 2. 
Epidemiology and etiology. Schizophr Res. 2008;102(13):1-18. doi:10.1016/j.schres.2008.04.011.

12. Guloksuz S, van Os J. The slow death of the concept of schizophrenia and the painful birth of the psychosis spectrum. 2017. doi:10.1017/S0033291717001775.

13. Insel TR, Cuthbert BN. Brain disorders? Precisely.

Science (80- ). 2015;348(6234):499-500.

doi: $10.1126 /$ science.aab2358.

14. Kapur S, Phillips AG, Insel TR. Why has it taken so long for biological psychiatry to develop clinical tests and what to do about it? Mol Psychiatry. 2012;17(12):11741179. doi:10.1038/mp.2012.105.

15. Epskamp S, Waldorp LJ, Mõttus R, Borsboom D. Discovering Psychological Dynamics: The Gaussian Graphical Model in Cross-sectional and Time-series Data. 2016:1-56. http://arxiv.org/abs/1609.04156.

16. Boschloo L, Van Borkulo CD, Borsboom D, Schoevers RA. A Prospective Study on How Symptoms in a Network Predict the Onset of Depression. Psychother Psychosom. 2016;85(3):183-184. doi:10.1159/000442001.

17. Fried EI, Epskamp S, Nesse RM, Tuerlinckx F, Borsboom D. What are "good" depression symptoms? Comparing the centrality of DSM and non-DSM symptoms of depression in a network analysis. J Affect Disord. 2015;189:314-320. doi:10.1016/j.jad.2015.09.005. 18. van de Leemput IA, Wichers M, Cramer AOJ, et al. Critical slowing down as early warning for the onset and termination of depression. Proc Natl Acad Sci.

2014;111(1):87-92. doi:10.1073/pnas.1312114110.

19. Fried EI, Bockting C, Arjadi R, et al. From Loss to Loneliness: The Relationship Between Bereavement and Depressive Symptoms. J Abnorm Psychol.

2015;124(2):256-265. doi:10.1037/abn0000028.

20. Bringmann LF, Lemmens LHJM, Huibers MJH, Borsboom D, Tuerlinckx F. Revealing the dynamic network structure of the Beck Depression Inventory-II. Psychol Med. 2015;45(4):747-757. doi:10.1017/S0033291714001809.

21. van Borkulo CD, Boschloo L, Borsboom D, Brenda WJHP, Waldorp LJ, Schoevers RA. Association of Symptom Network Structure With the Course of Depression. 2016;72(12):1219-1226.

doi:10.1001/jamapsychiatry.2015.2079.

22. Cramer AOJ, Borsboom D, Aggen SH, Kendler KS.

The pathoplasticity of dysphoric episodes: differential impact of stressful life events on the pattern of depressive symptom inter-correlations. Psychol Med. 2012;42(5):957965. doi:10.1017/S003329171100211X.

23. Fried EI, Nesse RM. The impact of individual depressive symptoms on impairment of psychosocial functioning. PLoS One. 2014;9(2):e90311.

doi:10.1371/journal.pone.0090311.

24. McNally RJ, Robinaugh DJ, Wu GWY, Wang L, Deserno M, Borsboom D. Mental Disorders as Causal Systems: A Network Approach to Posttraumatic Stress Disorder. Clin Psychol Sci. 2011:1-14.

doi:10.1177/2167702614553230.
25. Isvoranu AM, van Borkulo CD, Boyette LL, Wigman JTW, Vinkers CH, Borsboom D. A Network Approach to Psychosis: Pathways between Childhood Trauma and Psychotic Symptoms. Schizophr Bull. 2016;(Advance Access):1-10. doi:10.1093/schbul/sbw055.

26. Isvoranu A-M, Borsboom D, van Os J, Guloksuz S. A Network Approach to Environmental Impact in Psychotic Disorder: Brief Theoretical Framework. Schizophr Bull. 2016;42(4):870-873. doi:10.1093/schbul/sbw049.

27. Bak M, Drukker M, Hasmi L, Van Jim OS. An n=1 Clinical network analysis of symptoms and treatment in psychosis. PLoS One. 2016;11(9).

doi:10.1371/journal.pone.0162811.

28. van Rooijen G, Isvoranu AM, Meijer CJ, van Borkulo CD, Ruhé HG, de Haan L. A symptom network structure of the psychosis spectrum. Schizophrenia Research. 2016. 29. Deserno MK, Borsboom D, Begeer S, Geurts HM. Multicausal systems ask for multicausal approaches: A network perspective on subjective well-being in individuals with autism spectrum disorder. Autism. 2016. doi: $10.1177 / 1362361316660309$.

30. Ruzzano L, Borsboom D, Geurts HM. Repetitive behaviors in autism and obsessive-compulsive disorder: new perspectives from a network analysis. J Autism Dev Disord. 2015;45(1):192-202. doi:10.1007/s10803-0142204-9.

31. Beard C, Millner AJ, Forgeard MJC, et al. Network Analysis of Depression and Anxiety Symptom Relations in a Psychiatric Sample. Psychol Med. 2016;(2016):1-11. doi:10.1017/S0033291716002300.

32. Heeren A, McNally RJ. An integrative network approach to social anxiety disorder: The complex dynamic interplay among attentional bias for threat, attentional control, and symptoms. J Anxiety Disord. 2016;42:95-104. doi:10.1016/j.janxdis.2016.06.009.

33. Rhemtulla M, Fried EI, Aggen SH, Tuerlinckx F, Kendler KS, Borsboom D. Network analysis of substance abuse and dependence symptoms. Drug Alcohol Depend. 2016;161:230-237. doi:10.1016/j.drugalcdep.2016.02.005. 34. Cramer AOJ, van der Sluis S, Noordhof A, et al.

Dimensions of normal personality as networks in search of equilibrium: You can't like parties if you don't like people. Eur J Pers. 2012;26(4):414-431. doi:10.1002/per.1866.

35. Costantini G, Epskamp S, Borsboom D, et al. State of the $\{\mathrm{aRt}\}$ personality research: $\{\mathrm{A}\}$ tutorial on network analysis of personality data in $\{\mathrm{R}\}$. J Res Pers. 2015;54:13-29. doi:10.1016/j.jrp.2014.07.003.

36. Kossakowski JJ, Epskamp S, Kieffer JM, Borkulo CD, Rhemtulla M, Borsboom D. The application of a network approach to Health-Related Quality of Life (HRQoL): introducing a new method for assessing HRQoL in healthy adults and cancer patients. Qual Life Res. 2015;25(4):781792. doi:10.1007/s11136-015-1127-z.

37. Tio P, Epskamp S, Noordhof A, Borsboom D. Mapping the manuals of madness: Comparing the ICD-10 and DSM-IV-TR using a network approach. Int J Methods 
Psychiatr Res. 2016;25(4):267-276.

doi:10.1002/mpr.1503.

38. Borsboom D, Cramer AOJ, Schmittmann VD, Epskamp S, Waldorp LJ. The Small World of

Psychopathology. PLoS One. 2011;6(11).

doi:10.1371/journal.pone.0027407.

39. Boschloo L, Schoevers RA, van Borkulo CD,

Borsboom D, Oldehinkel AJ. The network structure of psychopathology in a community sample of preadolescents. J Abnorm Psychol. 2016;125(4):599-606. doi:10.1037/abn0000150.

40. Fried, E.I \& Cramer AOJ. Moving forward: Challenges and directino for psychopathological network theory and methodology. Perspect Psychol Sci. 2017.

doi:10.17605/OSF.IO/BNEK.

41. Scheffer M, Bascompte J, Brock WA, et al. Earlywarning signals for critical transitions. Nature. 2009;461(7260):53-59. doi:10.1038/nature08227. 42. Epskamp S, Cramer AOJ, Waldorp LJ, Schmittmann VD, Borsboom D. qgraph: Network Visualizations of Relationships in Psychometric Data. J Stat Softw. 2012;48(4):1-18. http://www.jstatsoft.org/v48/i04. 43. Frewen PA, Allen SL, Lanius R a, Neufeld RWJ. Perceived causal relations: novel methodology for assessing client attributions about causal associations between variables including symptoms and functional impairment. Assessment. 2012;19(4):480-493. doi:10.1177/1073191111418297.

44. Kim NS. Clinical psychologists' theory-based representations of mental disorders affect their diagnostic reasoning and memory. Diss Abstr Int Sect B Sci Eng. 2002;63(3-B):1565.

45. I.I. G. Schizophrenia and genetics. A Twin Study Vantage Point. ACADPRESS, NEW YORK, NY. 1972. 46. Leask SJ. Environmental influences in schizophrenia: the known and the unknown. Adv Psychiatr Treat. 2004;10(5):323-330. doi:10.1192/apt.10.5.323. 47. van Os J, Kapur S. Schizophrenia. Lancet. 2009;374(9690):635-645. doi:10.1016/S01406736(09)60995-8.

48. van Os J, Kenis G, Rutten BPF. The environment and schizophrenia. Nature. 2010;468(7321):203-212. doi:10.1038/nature09563.

49. Tsuang MT, Stone WS, Faraone S V. Genes, environment and schizophrenia. Br J Psychiatry. 2001;178(SUPPL. 40). doi:10.1192/bjp.178.40.s18. 50. Fried EI, Nesse RM, Zivin K, Guille C, Sen S. Depression is more than the sum score of its parts: individual DSM symptoms have different risk factors. Psychol Med. 2014;44(10):2067-2076. doi:10.1017/S0033291713002900.

51. Kay SR, Fiszbein A, Opler LA. The Positive and Negative Syndrome Scale (PANSS) for Schizophrenia. Schizophr Bull. 1987;13(2):261-276.

doi:10.1093/schbul/13.2.261.

52. Guloksuz S, Nierop M Van, Lieb R, Winkel R Van, Wittchen H, Os J Van. Evidence that the presence of psychosis in non-psychotic disorder is environmentdependent and mediated by severity of non-psychotic psychopathology. Psychol Med. 2015:1-13. doi:10.1017/S0033291715000380.

53. Guloksuz S, van Nierop M, Bak M, et al. Exposure to environmental factors increases connectivity between symptom domains in the psychopathology network. BMC Psychiatry. 2016;16(1):223. doi:10.1186/s12888-0160935-1.

54. Fusar-Poli P, Nelson B, Valmaggia L, Yung AR, McGuire PK. Comorbid depressive and anxiety disorders in 509 individuals with an at-risk mental state: Impact on psychopathology and transition to psychosis. Schizophr Bull. 2014;40(1):120-131. doi:10.1093/schbul/sbs136. 55. Smeets F, Lataster T, Viechtbauer W, et al. Evidence that environmental and genetic risks for psychotic disorder may operate by impacting on connections between core symptoms of perceptual alteration and delusional ideation. Schizophr Bull. 2015;41(3):687-697.

doi:10.1093/schbul/sbu122.

56. Wiersma JE, Hovens JGFM, Van Oppen P, et al. The importance of childhood trauma and childhood life events for chronicity of depression in adults. J Clin Psychiatry. 2009;70(7):983-989. doi:10.4088/JCP.08m04521.

57. Heim C, Nater UM, Maloney E, Boneva R, Jones JF, Reeves WC. Childhood trauma and risk for chronic fatigue syndrome: association with neuroendocrine dysfunction. Arch Gen Psychiatry. 2009;66(1):72-80.

doi:10.1001/archgenpsychiatry.2008.508.

58. Dannlowski U, Stuhrmann A, Beutelmann V, et al. Limbic scars: long-term consequences of childhood maltreatment revealed by functional and structural magnetic resonance imaging. Biol Psychiatry. 2012;71(4):286-293. doi:10.1016/j.biopsych.2011.10.021. 59. Read J, Van Os J, Morrison AP, Ross CA. Childhood trauma, psychosis and schizophrenia: A literature review with theoretical and clinical implications. Acta Psychiatr Scand. 2005;112(5):330-350. doi:10.1111/j.16000447.2005.00634.x.

60.Patton GC, Coffey C, Carlin JB, Degenhardt L, Lynskey M, Hall W. Cannabis use and mental health in young people: cohort study. BMJ. 2002;325(7374):11951198. doi:10.1136/bmj.325.7374.1195.

61. Bovasso GB. Cannabis abuse as a risk factor for depressive symptoms. Am J Psychiatry. 2001;158(12):2033-2037. doi:10.1176/appi.ajp.158.12.2033.

62. Arseneault L, Cannon M, Poulton R, Murray R, Caspi A, Moffitt TE. Cannabis use in adolescence and risk for adult psychosis: longitudinal prospective study. $B M J$. 2002;325(7374):1212-1213. doi:10.1136/bmj.325.7374.1212. 63. Kroeze R, van der Veen D, Servaas M, et al. Personalized feedback on symptom dynamics of psychopathology: A proof-of-principle study. J Pers Res. 2017;3(1):1-10. doi:10.17505/jpor.2017.01. 
64. Moritz S, Woodward TS. Metacognitive training in schizophrenia: from basic research to knowledge translation and intervention. Curr Opin Psychiatry. 2007;20(6):619-625. doi:10.1097/YCO.0b013e3282f0b8ed.

65. Hayes SC, Strosahl KD, Wilson KG. Acceptance and Commitment Therapy: An Experiential Approach to Behavior Change.; 1999.

66. Bringmann LF, Vissers N, Wichers M, et al. A network approach to psychopathology: new insights into clinical longitudinal data. PLoS One. 2013;8(4):e60188.

doi:10.1371/journal.pone.0060188 [doi] $\backslash$ rPONE-D-1229830 [pii].

67. Epskamp S, van Borkulo CD, van der Veen D, Isvoranu AM, Riese H, Cramer AOJ. Personalized
Network Modeling in Psychopathology: The Importance of Contemporaneous and Temporal Connections. Clin Psychol Sci. 2017; submitted.

68. Fisher AJ, Boswell JF. Enhancing the Personalization of Psychotherapy With Dynamic Assessment and Modeling. Assessment. 2016;23(4):496-506. doi:10.1177/1073191116638735.

69. Guloksuz S, Pries L-K, van Os J. Application of network methods for understanding mental disorders: pitfalls and promise. Psychol Med. 2017:1-10. doi:10.1017/S0033291717001350.

70. R Development Core Team. A Lang Environ Stat Comput. 2015;55:275-286. 